\title{
Structure-Function Changes of the Distal Outflow Tract in Response to Nitric Oxide
}

Susannah Waxman ${ }^{1}$, Chao Wang ${ }^{1,2,3}$, Yalong Dang ${ }^{1}$, Ying Hong ${ }^{1,4}$, Hamed Esfandiari ${ }^{1}$, Priyal Shah ${ }^{1}$, Kira L. Lathrop ${ }^{1}$, Ralitsa T. Loewen ${ }^{1}{ }^{*}$ Nils A. Loewen ${ }^{1}$

1: Department of Ophthalmology, University of Pittsburgh Medical Center, Pittsburgh, Pennsylvania, United States

2: Department of Ophthalmology, Xiangya Hospital, Central South University, Changsha, Hunan, China.

3: The Third Xiangya Hospital of Central South University, Changsha, Hunan, China.

4: Department of Ophthalmology, Peking University Third Hospital, Beijing, China.

* corresponding author 


\begin{abstract}
Purpose: To correlate outflow function and outflow tract vessel diameter changes induced by nitric oxide (NO).

Methods: In a porcine anterior segment perfusion model, the effects of a nitric oxide donor (100 $\mu$ M DETANO) on outflow facility were compared to controls ( $n=8$ per group) with trabecular meshwork (TM) and after circumferential ab interno trabeculectomy (AIT). Outflow structures were assessed with spectral domain optical coherence tomography (SD-OCT) before and after NO, or an NO synthase inhibitor (100 $\mu \mathrm{M}$ L-NAME) and the vasoconstrictor, endothelin-1 (100 pg/mL ET-1). Scans were processed with a custom macro script and aligned for automated reslicing and quantification of cross-sectional outflow tract areas (CSA).
\end{abstract}

Results: The facility increased after DETA-NO $(0.189 \pm 0.081 \mu \mathrm{L} / \mathrm{min} \cdot \mathrm{mmHg}, \mathrm{p}=0.034)$ and AIT $(0.251 \pm 0.094$ $\mu \mathrm{L} / \mathrm{min} \cdot \mathrm{mmHg}, \mathrm{p}=0.009)$, respectively. Even after AIT, DETA-NO increased the facility by $61.5 \%$ $(0.190 \pm 0.074 \mu \mathrm{L} / \mathrm{min} \cdot \mathrm{mmHg}, \mathrm{p}=0.023)$ and CSA by $13.9 \%(p<0.001)$. L-NAME + ET-1 decreased CSA by $-8.6 \%$ $(p<0.001)$. NO increased the diameter of focal constrictions $5.0 \pm 3.8$ fold.

Conclusions: NO can dilate vessels of the distal outflow tract and increase outflow facility in a TMindependent fashion. There are short, focally constricting vessel sections that display large diameter changes and may have a substantial impact on outflow.

Keywords: glaucoma; nitric oxide; spectral domain optical coherence tomography; aqueous humor outflow tract; physiology 


\section{Introduction}

Although the trabecular meshwork (TM) has long been considered the primary site of outflow resistance, its surgical removal or bypass does not lower the intraocular pressure (IOP) to the predicted level of episcleral venous pressure. ${ }^{1-3}$ Recent laboratory studies showed that about $50 \%$ of outflow resistance is located further downstream. ${ }^{4,5}$ Our clinical studies of plasma-mediated ab interno trabeculectomy (AIT) show that the outflow resistance distal to the TM is higher in eyes with glaucoma. ${ }^{6-9}$ Only a small fraction of patients (about $0.3 \%$ ) achieve the expected intraocular pressure (IOP). ${ }^{9}$ In fact, an empirical formula predicts that patients cannot achieve an IOP lower than $18.6 \mathrm{mmHg}$ without additional aqueous suppressants ${ }^{10}$ resulting in a failure rate of up to $30 \%$ within 12 months ${ }^{6}$ for a target below 12 $\mathrm{mmHg}$ in moderate to severe glaucoma. The pre- and post-operative IOP in AIT are correlated, ${ }^{6}$ indicating an increased post-TM outflow resistance in eyes with a higher IOP. This suggests an incomplete understanding of outflow distal to the TM, and an avenue for new, targeted therapies. To explain the outflow resistance with the known numbers and diameters of distal outflow tract vessels, ${ }^{11,12}$ it has been speculated that not all outflow channels may be patent at the same time, or they could constrict and dilate. ${ }^{5,13,14}$ In theory, minute changes of small vessels, like collector channels (CC), could profoundly influence the facility. To generate the outflow resistance, they would have to have a diameter of only 20 $\mu \mathrm{m}$ yet most have a diameter of about $50 \mu \mathrm{m}$ or larger. A single vessel of this diameter could carry the entire flow, ${ }^{5}$ a principle applied to ab interno micro gel stents to allow for a slow and safe aqueous humor drainage from the anterior chamber to the subconjunctival space. ${ }^{15} \mathrm{CC}$ diameter changes from IOP variations ${ }^{16}$ and with the cardiac pulse wave ${ }^{17}$ do not explain the remaining distal outflow resistance, nor do valves at the orifices of collector channels ${ }^{18}$ as their removal by deep sclerotomy fails to reduce IOP further. ${ }^{19,20}$

In this study, we hypothesized that NO, an established hypotensive agent, can increase the facility in a TM-independent manner by dilating outflow tract vessels. We investigated its effect with and without circumferential AIT, developed an automated, quantitative, and live analysis of the cross-sectional area (CSA) of intrascleral outflow vessels, and searched for evidence of focal dilation and constriction that may show a reactive outflow regulatory mechanism.

\section{Materials and methods}

\section{Study design}

This study was designed as described in the following and detailed below. We used pilot eyes to establish that $360^{\circ}$ AIT was a viable way to remove TM and increase facility. In experiment 1 , eyes with intact TM were perfused at $4 \mu \mathrm{L} / \mathrm{min}$, a high normal rate to elevate make a facility change and pressure reduction more discoverable by increasing the testing power. In group NO, media was supplemented with DETA-NO while controls (C) went through the same steps but with standard media (NO: $n=8, C: n=8$ ). In experiment 2, eyes with TM removed circumferentially by AIT were perfused at $6 \mu \mathrm{L} / \mathrm{min}$, in order to make a facility change easier to detect after a significant portion of the outflow resistance has been eliminated. Eyes in AIT-NO ( $n=8)$ received DETA-NO supplemented media while controls (AIT-C, $n=8$ ) went through the same steps but were perfused with conventional media. In experiment 3, outflow tract vessel dilation was quantified using wide-spectrum spectral domain optical coherence tomography (SD-OCT) and received 
either NO-supplemented perfusion media $(\mathrm{NO}, \mathrm{n}=3)$ or L-NAME + ET-1-supplemented media (L-NAME + ET$1: n=3)$.

\section{Anterior segment perfusion culture}

Porcine eyes were acquired on the day of experiments from a local abattoir (Thoma Meat Market, Saxonburg, Pittsburgh PA) and prepared for perfusion culture within two hours of sacrifice as detailed previously. ${ }^{21-24}$ In brief, extraocular tissues and conjunctiva were trimmed away from globes. In an aseptic hood, globe exteriors were decontaminated via submersion in ophthalmic $5 \%$ betadine solution (NC9771653, Fisher Scientific, Waltham, Massachusetts, United States) for two minutes and rinsed three times with phosphate buffered saline (PBS, 14080-055, Fisher Scientific, Fisher Scientific, Waltham, Massachusetts, United States). After hemisecting them, the choroid, iris, and ciliary body were carefully removed. The anterior segments were mounted on custom perfusion dishes, anterior chambers were filled with perfusion media (Dulbecco's modified Eagle medium (DMEM, sh30284.02, Fisher Scientific, Waltham, Massachusetts, United States), 1\% FBS (Fisher Scientific, 10082-147), 1\% antibiotic, antimycotic (Fisher Scientific, 15240-062)) and perfused with a microinfusion pump (70-3007, Harvard Apparatus, Holliston, Massachusetts, United States). The eyes were cultured at $37^{\circ} \mathrm{C}$ with $5 \%$ atmospheric $\mathrm{CO}_{2}$ and perfused for at least 48 hours to acclimate and establish a stable baseline. A humidity pan was not used. IOPs were measured at two-minute intervals with pressure transducers (Deltran II: DPT-200, Utah Medical Products, Midvale, Utah, United States) and recorded (FE224, PL3508/P, MLA1052, ADInstruments, Sydney, Australia) and analyzed (LabChart 7, ADInstruments, Sydney, Australia). Cultures treated with NO received media supplemented with $100 \mu \mathrm{M}$ diethylenetriamine nitric oxide adduct (DETA-NO, D185, Sigma-Aldrich, St. Louis, Missouri, United States), an NO donor, prepared from powder on the same day of experiments. Due to a 57 hour half-life of DETA-NO in solution at room temperature and a 23 -hour half-life at $37^{\circ} \mathrm{C}^{25}$ fresh media was exchanged in syringes every 48 hours. Eight eyes were perfused in parallel for seven days and randomly assigned to treatment groups.

\section{Statistical analysis}

Facility changes were analyzed using a mixed effect mode ${ }^{26}$ in $\mathrm{R}^{27}$ Averages were derived from three-hour periods. Models were fitted using the Imer function in the Ime4 package ${ }^{28}$ with fixed effects for treatment and random effects for subject and time-point. After TM ablation, the post-surgical data was compared to baseline with a student's t-test in PASW 18.0 software (SPSS Inc., Chicago, Illinois, United States). All values were reported as the mean and standard error of the mean (mean \pm SEM) unless otherwise stated to show the uncertainty around the estimate of the mean measurement. ${ }^{29}$ After media changes, recordings for the next six hours were excluded from analysis. Data are graphically presented as the mean facility change ( $\Delta$ facility) over time. A p-value less than or equal to 0.05 was considered statistically significant.

\section{Volumetric wide-spectrum spectral domain optical coherence tomography}

Anterior segments were dissected as previously described. Segments were mounted on perfusion dishes, and gravity perfused for 30 minutes with perfusion media at the water column height equivalent of $15 \mathrm{mmHg}$. Perfusion dishes were affixed to the center of a rotatable stage (XYR1, ThorLabs, Newton, New Jersey, United States; Supplementary Fig. 1) and placed under a $10 \mathrm{~mm}$ telecentric lens attached to the 
sample arm of a spectral domain optical coherence tomography system (SD-OCT, Envisu R2210, Leica (Bioptigen), Morrisville, North Carolina, United States). The sample arm was held in place with the adjustable carriage for a dissecting microscope, positioned so that the scanning beam was oriented perpendicularly to the limbus, and adjusted to focus on the tissue. The limbus was observed in the software's scan mode (InVivoVue, Bioptigen, Morrisville, North Carolina, United States). The stage was rotated to locate a region in each eye where volumetric scans of at least $60^{\circ}$ of consecutive limbus could be visualized. This included a small amount of cornea as a location reference, the distal sclera, and the signal voids of its corresponding vasculature in each scan. Scanned areas were $6 \mathrm{~mm}$ long (parallel to the limbus), $4 \mathrm{~mm}$ wide (cornea to distal sclera), and $1.6 \mathrm{~mm}$ deep. Due to the teardrop shape of the porcine cornea, the eye could not be rotated $360^{\circ}$ around a stationary axis while viewing the limbus in a $4 \mathrm{~mm}$ wide window. Eyes were rotated $20^{\circ}$ between scans. Between three and five baseline scans were obtained for each eye, depending on visualization. All scans were captured with enhanced depth imaging. After baseline scans, perfusion media was supplemented with either $100 \mu \mathrm{M}$ DETA-NO $(n=3)$ or with $100 \mathrm{pg} / \mathrm{mL}$ ET-1 and $100 \mu \mathrm{M}$ L-NAME $(n=3)$ and an anterior chamber exchange was performed. After 40 minutes of gravitybased perfusion, scans were taken with the identical parameters and in the same regions.

\section{Image processing}

SD-OCT images were processed in Image ${ }^{30}$ (Version 1.50i, National Institute of Health, Bethesda, Maryland, United States) and Amira Aviso (version 9.1, FEl, ThermoScientific, Waltham, Massachusetts) to remove noise, align pre-and post-treatment outflow tract signal voids in a three-dimensional space (3D), and to allow automated, quantitative measurement of cross-sectional areas (CSA). The SD-OCT files were converted to 8-bit TIFF stacks in ImageJ and cleaned with a custom ImageJ macro script to denoise and extract signal voids from stacks. A Kalman Stack Filter with an acquisition noise estimate of 0.05 and bias placed on the prediction of 0.80 was employed to reduce salt-and-pepper noise inherent to SD-OCT imaging, using oversampling in the XY direction. Grayscale Inversion was used to convert dark signal voids into bright structures while highly-scattering sclera was converted into dark areas. The Subtract Background function with a rolling ball radius of 100 pixels was used to reduce signal not encapsulated by the sclera. A uniform threshold with a brightness value cutoff of 64 was applied to each frame of each volume. A series of 3D erosions was employed to remove solitary and small islands of white pixels often too small to be structures of interest, further reducing speckle noise, and equal 3D dilation was used to return subtracted pixels to the remaining structures. Stacks were resliced, avoiding interpolation, so that 2D images were visualized along the more conventionally analyzed sagittal plane as opposed to along the transverse B-scans captured. Binary image sequences were saved as 8-bit TIFF stacks.

Minimally processed versions of stacks underwent the grayscale inversion, background subtraction, and reslicing steps only. These functions were chosen to aid in visualization of signal voids without the more aggressive denoising conferred by the complete macro script process. Cleaned and minimally processed stacks from both pre- and post-treatment scans of the same region were imported into Amira, voxels in each dimension were calibrated to true size in microns, and volumes were down-sampled to $600 \mathrm{x}$ $600 \times 600$ voxel volumes through a Lanczos filter with a Resample module to reduce computational burden of subsequent processing. Outflow structures were rendered in a three-dimensional virtual space (3D) with a Volume rendering module. 3D alignment of pre- and post-treatment volumes was performed in Amira by 
linking the two cleaned, resampled images with the Register module and selecting the Rigid transformation option before execution. Volumes were visualized during automatic alignment to ensure registration was successful and that pre-and post-treatment scans colocalized. If volumes did not show colocalization of outflow tract vessels and branching-point landmarks, the model dataset was repositioned to provide an approximation of alignment with its reference volume before rerunning automatic registration. Spatial coordinates of aligned, cleaned data were conferred to their respective minimally processed scans to align them. The upper and lower-bound color map thresholds of minimally processed data were adjusted to best resolve outflow structures.

Aligned, cleaned data were resampled with their transformed spatial coordinates with the Resample Transformed Image module, and the Multi-Thresholding tool was used to create a label field that included white voxels of each volume and excluded black ones. Any signal from the corneal region or above the bounds of the sclera was removed in Segmentation mode with the 3D lasso tool in the same regions of both pre-and post-treatment scans. Label fields for pre-treatment scans were pseudo-colored in red, and post-treatment scans were pseudo-colored in cyan. Surfaces were generated with extended bounding boxes and a constrained smoothing factor of 5 from each label field. Scan Surface to Volume modules were created for each the pre-and post-treatment surfaces, and Bounding box size and Dimensions fields were linked between the two scans via the Connection Editor. Surfaces were scanned to volumes with identical bounding box sizes and coordinates. Extract Subregion modules were attached to each resulting volume, Box Min and Box Size between scans were linked. The boundaries of the extraction area were adjusted over the samples to isolate only the region from both volumes captured in both scans after alignment to ensure that changes in CSA values were attributed to vasoreactivity and not to artifacts. A Material Statistics module was attached to the extracted subregions and Area per slice was selected for calculation.

The resulting spreadsheet output contained CSAs of visualized structures along the 600 (or fewer, depending on the extent of subregion cropping) images in each scan. Statistical results were confirmed with the use of an Ortho Slice module to view cross-sections of the extracted subregions. This ensured that preand post-treatment cross-sections were taken of the same locations in each scan, that signal voids appeared similar in 2D shape and location between pre-and post-treatment cross-sections, and that differences in CSA values corresponded with differences in visually appreciated CSAs. The slice number chosen along the translation bar in the Ortho Slice properties panel corresponded with slice location IDs and respective CSA values in the Amira output. Five individual orthogonal slices were created with the Extract Image module and exported as 2D TIF files. CSA calculation according to the Amira output was evaluated in ImageJ for cross-platform confirmation. Total values of pre-and post-treatment CSAs were compared by paired t-test.

\section{Results}

\section{Outflow facility increase by NO before and after TM ablation}

In cultures with intact TM, baseline facilities in NO and $\mathrm{C}$ were not different $(\mathrm{p}=0.80)$. Posttreatment facility of $\mathrm{C}$, with a mean decrease of $0.087 \pm 0.010 \mu \mathrm{L} / \mathrm{min} \cdot \mathrm{mmHg}$, was compared to NO, with a mean increase of $0.113 \pm 0.011 \mu \mathrm{L} / \mathrm{min} \cdot \mathrm{mmHg}$, through a mixed-effects model, indicating a significant NOmediated facility increase of $0.189 \pm 0.081 \mu \mathrm{L} / \mathrm{min} \cdot \mathrm{mmHg}(46.83 \%, \mathrm{p}=0.034, \mathrm{NO}: \mathrm{n}=8, \mathrm{C}: \mathrm{n}=8$, Fig. 1$)$. 
Circumferential ablation of TM induced a significant facility increase $(0.251 \pm 0.160,59.2 \%)$ from baseline $(p<0.001)$. Eyes were perfused before and after AIT, in groups AIT-NO and AIT-C as in our prior experiments. ${ }^{31-35}$ One eye had to be excluded due to contamination (N: AIT-NO=7, AIT-C=8). Baseline facility measures in AIT-NO and AIT-C were not different ( $p=0.37$ ). Post-treatment facilities for AIT-C, with a mean change of $-0.055 \pm 0.009 \mu \mathrm{L} / \mathrm{min} \cdot \mathrm{mmHg}$, showed a slight and steady decrease throughout the experiment while facilities in AIT-NO, with a mean change of $0.179 \pm 0.021 \mu \mathrm{L} / \mathrm{min} \cdot \mathrm{mmHg}$, were increased from baseline at all post-treatment time-points. After AIT, a mixed-effects model showed a significant NOmediated facility increase of $0.190 \pm 0.074 \mu \mathrm{L} / \mathrm{min} \cdot \mathrm{mmHg}(61.49 \% \mathrm{p}=0.023$, Fig. 2).

\section{Volumetric SD-OCT scanning and image processing}

Limbal outflow structures were visualized successfully. A contrast agent was not necessary. Gravity perfusion was used for these experiments to allow for rapid yet gentle pressurization and fluid exchange. Previous experiments indicated a flow rate of $3.7 \pm 1.6 \mu \mathrm{l} / \mathrm{min}$ at a constant gravity perfusion with a physiologic pressure of $15 \mathrm{mmHg}^{36}$ equivalent to $20.3 \mathrm{~cm}$ water column. ${ }^{21}$ Volumetric SD-OCT scans were captured at a minimum of three adjacent locations for each eye. A single scan required 21 seconds. Image J processing of SD-OCT volumes with the custom macro script could isolate intrascleral signal voids within outflow tract vessels of the same regions shown in corresponding, unprocessed B-scans. Compared to previous grayscale inversion and background subtraction alone, there was reduced noise and minimal loss of signal. Scans could be manipulated in a virtual 3D space to view structures from all angles and showed good agreement with outflow tract vessels visible in confocal microscopy. ${ }^{12}$ Compared to minimally processed scans (Fig. 3 B1-5), features of structures were well preserved in cleaned scans with sufficient noise reduction (Fig. 3 C1-5).

\section{NO induces outflow vessel dilation and releases focal restrictors}

CSA values for each 3D rendering could be automatically quantified and reported by the software along with the corresponding cross-section location. As seen in 3D renderings of scans from a representative DETA-NO treated eye (Fig. 4 A-C 0-40 ), a larger wrapping with cyan in most locations corresponded with higher CSA values in the corresponding plots below (Fig. 8C 0-40 ). Conversely, in the 3D renderings of a representative L-NAME+ ET-1 treated eye (Fig. $4 C, D 0-40^{\circ}$ ), more extensive coverage of pretreatment (red) in most areas corresponded with higher overall CSA values in the corresponding plots below (Fig. 4D-F 0-40 ). Post-treatment CSA was compared to pre-treatment baseline in of each three eyes treated with DETA-NO (22 scans total, 13,146 virtual sections) and three eyes treated with L-NAME+ ET-1 (18 scans total, 10,580 virtual sections). DETA-NO increased CSA by an average of $13.9 \%$ and L-NAME+ ET-1 decreased CSA by an average of $8.6 \%$ (Fig. 5, p $<0.001$ for both groups). CSAs from each eye demonstrated the expected trend (Supplementary Table 1). CSA calculation according to the Amira output was verified in ImageJ for five slices, and CSA values corresponded precisely.

Besides quantitative evaluation, structural changes of outflow tract volume could be appreciated in 3D. In NO (Fig. 6), outflow tract vessels appeared to open closed locations (white arrows), release focal restrictors at sites of high response (black arrows), and dilate throughout. In contrast, outflow tract vessels in L-NAME+ ET-1 (Fig. 7) constricted or collapsed at sites of high response (black arrows). Focal restrictors had a diameter increase to $502 \pm 376 \%$ after NO (Fig. 6). Conversely, L-NAME+ ET-1 caused a focal constriction to $53 \pm 9 \%$ (Fig. 7). While cleaning removed visual noise at the expense of removing the signal 
from more delicate structures, minimally processed scans (Fig. 6-7, A1-B4) showed 3D patterns similar to the cleaned data used for quantitative slice-by-slice analysis (Fig 6-7, C1-D4).

\section{Discussion}

This study determined functional and structural effects of NO on the conventional outflow tract distal to the TM. We found that NO, a messenger and hypotensive compound, increased the facility even after circumferential AIT. Our work agrees with McDonnell et al.'s recent study which describes the ability of DETA-NO to acutely increase facility even after TM removal not only in porcine but also in human eyes. ${ }^{37}$ Here, we confirmed those findings and extended the analysis by showing a facility change for up to five days. We showed that vasodilation of outflow vessels can be directly observed by SD-OCT including focal flow restrictors and vessels small enough to impact the facility, as theorized to exist.

Because unconjugated NO has a half-life of several seconds, ${ }^{38}$ we chose DETA-NO due to its relative stability compared to other NO-donors with more rapid NO release. The hypotensive effects of DETA-NO have been established in both porcine and human anterior segment perfusion culture, ${ }^{39,40}$ including after TM removal. ${ }^{37}$ Because of the short time from recovery to culture, we did not have to use a NO synthase (NOS) inhibitor or precontraction agent as may be necessary in human donor eyes with variable freshness to observe a hypotensive effect. ${ }^{37} \mathrm{TM}$ ablation by AIT was performed over the entire circumference in this study to create direct access to the collector channels and eliminate TM-based outflow resistance.

Consistent with our prior experience, ${ }^{21,23,24,34,41,42}$ the perfused porcine anterior segments did not experience the amount of corneal edema reported with other culture techniques. ${ }^{43}$ With intact TM, nitric oxide increased the facility by approximately $45 \%$ in group NO compared to the control group, C. After $360^{\circ}$ AIT in experiment 2 , we found a facility difference of approximately $60 \%$ between AIT-NO and AIT-C, in line with the findings reported by McDonnell et al. ${ }^{37}$ This difference persisted throughout experiments 1 and 2 but declined toward the end of experiment 1, possibly due to a decreasing TM facility response to NO. The TM makes for a significant contribution to outflow resistance, but not in experiment 2 where the facility enhancement is TM-independent. Trabectome-mediated TM ablation results in an approximately 300 micron-wide excision down to the bare sclera $24,31-34,41,44$ where the opening of the collector channels are located without significant trauma or thermal injury. Like DETA-NO, cromakalim treatment of mouse eyes lowered episcleral venous pressures, and in TM-free human anterior segment cultures, lowered IOP. ${ }^{45}$ These results suggest a target for pharmaceutical manipulation of post-trabecular outflow resistance that may be especially relevant after microincisional surgeries that remove or bypass the TM.

We examined the outflow tract vessels directly with SD-OCT which allowed us to obtain fullthickness image stacks of the perilimbal sclera. ${ }^{46-48}$ We improved this method by automating segmentation and assembly for a faster and more objective process. It was possible to isolate signal voids and reconstruct them into a complex outflow tract vessel network without the need to fix and clear this tissue as we have done before, ${ }^{12}$ but at the cost of a lower resolution. While most features were preserved after processing scans, it is important to recall that any denoising can cause loss of details of the structure. Confocal microscopy can produce high resolution volumes as well, but in order to reach comparable imaging depth, tissue must be fixed and cleared. Higher resolution imaging of living tissue can also be achieved with twophoton microscopy, but the imaging depth is more limited and could not be used here. ${ }^{49}$ 
Automated reconstruction of the outflow tract from SD-OCT signal voids allowed direct observation of vasomotion in vessels as large as $200 \mu \mathrm{m}$ and as small as $20 \mu \mathrm{m}$ in diameter. NO induced extensive vasodilation throughout the distal outflow tract while L-NAME + ET-1 caused substantial constriction. While these diameter changes alone might alter the outflow resistance, we observed many focal vessel segments that constrict and dilate significantly more. They may be the anatomic correlate for the rather dynamic outflow patterns observed in human patients and non-human primates. ${ }^{50}$ Smooth muscles surrounding collector channels, ${ }^{49}$ and NOS expression by SC and CC endothelium, ${ }^{51,52}$ suggest a site-specific capacity for distal outflow tract caliber regulation and segmental flow changes. ${ }^{13,53}$ Recently introduced NO-donating prostaglandin analogs $s^{54,55}$ may help address the lower levels of NO in eyes of primary open angle glaucoma patients and an overlooked, post-trabecular cause of reduced facility. ${ }^{56-58}$

In conclusion, while the TM is recognized as a principal site of conventional outflow resistance and regulation, we show that NO increases the outflow facility for as long as five days, both with intact TM and after circumferential AIT. NO dilates distal outflow tract vessels while L-NAME + ET-1 constricts them. Fivefold focal caliber changes can be observed.

\section{Acknowledgments}

Supported by National Eye Institute Grant K08EY022737 (NAL), Initiative to Cure Glaucoma of the Eye and Ear Foundation of Pittsburgh (NAL), Wiegand Fellowship of the Eye and Ear Foundation of Pittsburgh (YD), the Core Grant for Vision Research National Eye Institute Grant P30-EY08099, and a department grant by Research to Prevent Blindness (NAL).

\section{Disclosures}

S. Waxman, None; C. Wang, None; Y. Dang, None; Y. Hong None; H. Esfandiari, None; P. Shah, None; K.L. Lathrop, None; R.T. Loewen, None; N.A. Loewen, None 


\section{References}

1. Grant WM. Experimental aqueous perfusion in enucleated human eyes. Arch Ophthalmol. 1963;69:783-801.

2. Mäepea O, Bill A. Pressures in the juxtacanalicular tissue and Schlemm's canal in monkeys. Exp Eye Res. 1992;54(6):879-883.

3. Sit AJ, Ekdawi NS, Malihi M, McLaren JW. A novel method for computerized measurement of episcleral venous pressure in humans. Exp Eye Res. 2011;92(6):537-544.

4. Johnstone M. 3. Intraocular pressure control through linked trabecular meshwork and collector channel motion. In: Knepper PA, Samples JR, eds. Glaucoma Research and Clinical Advances 2016 to 2018. Kugler Publications; 2016:41.

5. Rosenquist R, Epstein D, Melamed S, Johnson M, Grant WM. Outflow resistance of enucleated human eyes at two different perfusion pressures and different extents of trabeculotomy. Curr Eye Res. 1989;8(12):1233-1240.

6. Bussel II, Kaplowitz K, Schuman JS, Loewen NA, Trabectome Study Group. Outcomes of ab interno trabeculectomy with the trabectome by degree of angle opening. Br J Ophthalmol. 2015;99(7):914919.

7. Loewen RT, Roy P, Parikh HA, Dang Y, Schuman JS, Loewen NA. Impact of a Glaucoma Severity Index on Results of Trabectome Surgery: Larger Pressure Reduction in More Severe Glaucoma. PLoS One. 2016;11(3):e0151926.

8. Bussel II, Kaplowitz K, Schuman JS, Loewen NA, Group TS, Others. Outcomes of ab interno trabeculectomy with the trabectome after failed trabeculectomy. Br J Ophthalmol. 2014;99(2):258262.

9. Parikh HA, Bussel II, Schuman JS, Brown EN, Loewen NA. Coarsened Exact Matching of PhacoTrabectome to Trabectome in Phakic Patients: Lack of Additional Pressure Reduction from Phacoemulsification. PLoS One. 2016;11(2):e0149384.

10. Neiweem AE, Bussel II, Schuman JS, Brown EN, Loewen NA. Glaucoma Surgery Calculator: Limited Additive Effect of Phacoemulsification on Intraocular Pressure in Ab Interno Trabeculectomy. PLoS One. 2016;11(4):e0153585.

11. Hann CR, Bentley MD, Vercnocke A, Ritman EL, Fautsch MP. Imaging the aqueous humor outflow pathway in human eyes by three-dimensional micro-computed tomography (3D micro-CT). Exp Eye Res. 2011;92(2):104-111.

12. Waxman S, Loewen RT, Dang Y, Watkins SC, Watson AM, Loewen NA. High-Resolution, ThreeDimensional Reconstruction of the Outflow Tract Demonstrates Segmental Differences in Cleared Eyes. Invest Ophthalmol Vis Sci. 2018;59(6):2371-2380.

13. Cha EDK, Xu J, Gong L, Gong H. Variations in active outflow along the trabecular outflow pathway. Exp Eye Res. 2016;146:354-360. 
14. Hann CR, Vercnocke AJ, Bentley MD, Jorgensen SM, Fautsch MP. Anatomic changes in Schlemm's canal and collector channels in normal and primary open-angle glaucoma eyes using low and high perfusion pressures. Invest Ophthalmol Vis Sci. 2014;55(9):5834-5841.

15. Sheybani A, Lenzhofer M, Hohensinn M, Reitsamer H, Ahmed IIK. Phacoemulsification combined with a new ab interno gel stent to treat open-angle glaucoma: Pilot study. J Cataract Refract Surg. 2015;41(9):1905-1909.

16. Xin C, Johnstone M, Wang N, Wang RK. OCT Study of Mechanical Properties Associated with Trabecular Meshwork and Collector Channel Motion in Human Eyes. PLoS One. 2016;11(9):e0162048.

17. Xin C, Wang RK, Song S, et al. Aqueous outflow regulation: Optical coherence tomography implicates pressure-dependent tissue motion. Exp Eye Res. 2017;158:171-186.

18. Johnstone MA. The aqueous outflow system as a mechanical pump: evidence from examination of tissue and aqueous movement in human and non-human primates. J Glaucoma. 2004;13(5):421-438.

19. Pajic B, Pajic-Eggspuehler B, Haefliger I. New minimally invasive, deep sclerotomy ab interno surgical procedure for glaucoma, six years of follow-up. J Glaucoma. 2011;20(2):109-114.

20. Singh D, Bundela R, Agarwal A, Bist HK, Satsangi SK. Goniotomy ab interno "a glaucoma filtering surgery" using the Fugo Plasma Blade. Ann Ophthalmol . 2006;38(3):213-217.

21. Loewen RT, Roy P, Park DB, et al. A Porcine Anterior Segment Perfusion and Transduction Model With Direct Visualization of the Trabecular Meshwork. Invest Ophthalmol Vis Sci. 2016;57(3):1338-1344.

22. Dang $\mathrm{Y}, \mathrm{Waxman} \mathrm{S}$, Wang $\mathrm{C}$, et al. Freeze-thaw decellularization of the trabecular meshwork in an ex vivo eye perfusion model. PeerJ. 2017;5:e3629.

23. Dang $\mathrm{Y}$, Loewen R, Parikh HA, Roy P, Loewen NA. Gene transfer to the outflow tract. Exp Eye Res. 2017;158:73-84.

24. Loewen RT, Brown EN, Roy P, Schuman JS, Sigal IA, Loewen NA. Regionally Discrete Aqueous Humor Outflow Quantification Using Fluorescein Canalograms. PLoS One. 2016;11(3):e0151754.

25. Keefer LK, Nims RW, Davies KM, Wink DA. "NONOates" (1-substituted diazen-1-ium-1,2-diolates) as nitric oxide donors: Convenient nitric oxide dosage forms. In: Methods in Enzymology. Vol 268. Academic Press; 1996:281-293.

26. Fan Q, Teo Y-Y, Saw S-M. Application of advanced statistics in ophthalmology. Invest Ophthalmol Vis Sci. 2011;52(9):6059-6065.

27. Core Team R. R: A Language and Environment for Statistical Computing. Vienna, Austria: R Foundation for Statistical Computing; 2016. http://www.R-project.org/.

28. Bates D, Mächler M, Bolker B, Walker S. Fitting Linear Mixed-Effects Models Using Ime4. Journal of Statistical Software, Articles. 2015;67(1):1-48.

29. Altman DG, Bland JM. Standard deviations and standard errors. BMJ. 2005;331(7521):903. 
30. Schindelin J, Arganda-Carreras I, Frise E, et al. Fiji: an open-source platform for biological-image analysis. Nat Methods. 2012;9(7):676-682.

31. Parikh HA, Loewen RT, Roy P, Schuman JS, Lathrop KL, Loewen NA. Differential Canalograms Detect Outflow Changes from Trabecular Micro-Bypass Stents and Ab Interno Trabeculectomy. Sci Rep. 2016;6:34705.

32. Loewen RT, Brown EN, Scott G, Parikh H, Schuman JS, Loewen NA. Quantification of Focal Outflow Enhancement Using Differential Canalograms. Invest Ophthalmol Vis Sci. 2016;57(6):2831-2838.

33. Wang C, Dang Y, Waxman S, Xia X, Weinreb RN, Loewen NA. Angle stability and outflow in dual blade ab interno trabeculectomy with active versus passive chamber management. PLoS One. 2017;12(5):e0177238.

34. Dang $Y$, Wang $C$, Shah $P$, et al. Outflow enhancement by three different ab interno trabeculectomy procedures in a porcine anterior segment model. Graefes Arch Clin Exp Ophthalmol. May 2018. doi:10.1007/s00417-018-3990-0

35. Dang $\mathrm{Y}$, Waxman $\mathrm{S}$, Wang $\mathrm{C}$, et al. Rapid learning curve assessment in an ex vivo training system for microincisional glaucoma surgery. Sci Rep. 2017;7(1):1605.

36. Ruiz-Ederra J, García M, Hernández M, et al. The pig eye as a novel model of glaucoma. Exp Eye Res. 2005;81(5):561-569.

37. McDonnell F, Dismuke WM, Overby DR, Stamer WD. PHARMACOLOGICAL REGULATION OF OUTFLOW RESISTANCE DISTAL TO SCHLEMM'S CANAL. Am J Physiol Cell Physiol. April 2018. doi:10.1152/ajpcell.00024.2018

38. Thomas DD, Liu X, Kantrow SP, Lancaster JR Jr. The biological lifetime of nitric oxide: implications for the perivascular dynamics of NO and O2. Proc Natl Acad Sci U S A. 2001;98(1):355-360.

39. Dismuke WM, Mbadugha CC, Ellis DZ. NO-induced regulation of human trabecular meshwork cell volume and aqueous humor outflow facility involve the BKCa ion channel. Am J Physiol Cell Physiol. 2008;294(6):C1378-C1386.

40. Ellis DZ, Dismuke WM, Chokshi BM. Characterization of soluble guanylate cyclase in NO-induced increases in aqueous humor outflow facility and in the trabecular meshwork. Invest Ophthalmol Vis Sci. 2009;50(4):1808-1813.

41. Dang $\mathrm{Y}, \mathrm{Waxman} \mathrm{S}$, Wang $\mathrm{C}$, Shah $\mathrm{P}$, Loewen $\mathrm{RT}$, Loewen NA. Intraocular pressure elevation precedes a phagocytosis decline in a model of pigmentary glaucoma. F1000Res. 2018;7. doi:10.12688/f1000research.13797.1

42. Dang $\mathrm{Y}$, Waxman $\mathrm{S}$, Wang $\mathrm{C}$, Loewen RT, Sun $\mathrm{M}$, Loewen NA. A porcine ex vivo model of pigmentary glaucoma. Sci Rep. 2018;8(1):5468.

43. Guindolet D, Crouzet E, He Z, et al. Storage of Porcine Cornea in an Innovative Bioreactor. Invest Ophthalmol Vis Sci. 2017;58(13):5907-5917.

44. Fallano K, Bussel I, Kagemann L, Lathrop KL, Loewen N. Training strategies and outcomes of ab interno 
trabeculectomy with the trabectome. F1000Res. 2017;6:67.

45. Roy Chowdhury U, Rinkoski TA, Bahler CK, et al. Effect of Cromakalim Prodrug 1 (CKLP1) on Aqueous Humor Dynamics and Feasibility of Combination Therapy With Existing Ocular Hypotensive Agents. Invest Ophthalmol Vis Sci. 2017;58(13):5731-5742.

46. Kagemann $L$, Wollstein $\mathrm{G}$, Ishikawa $\mathrm{H}$, et al. 3D visualization of aqueous humor outflow structures insitu in humans. Exp Eye Res. 2011;93(3):308-315.

47. Kagemann $L$, Wollstein $\mathrm{G}$, Ishikawa $\mathrm{H}$, et al. Visualization of the conventional outflow pathway in the living human eye. Ophthalmology. 2012;119(8):1563-1568.

48. Francis AW, Kagemann L, Wollstein G, et al. Morphometric Analysis of Aqueous Humor Outflow Structures with Spectral-Domain Optical Coherence Tomography SD-OCT Analysis of Aqueous Humor Outflow Structures. Invest Ophthalmol Vis Sci. 2012;53(9):5198-5207.

49. Gonzalez JM Jr, Ko MK, Hong Y-K, Weigert R, Tan JCH. Deep tissue analysis of distal aqueous drainage structures and contractile features. Sci Rep. 2017;7(1):17071.

50. Huang AS, Li M, Yang D, Wang H, Wang N, Weinreb RN. Aqueous Angiography in Living Nonhuman Primates Shows Segmental, Pulsatile, and Dynamic Angiographic Aqueous Humor Outflow.

Ophthalmology. 2017;124(6):793-803.

51. Chang JYH, Stamer WD, Bertrand J, et al. Role of nitric oxide in murine conventional outflow physiology. Am J Physiol Cell Physiol. 2015;309(4):C205-C214.

52. Nathanson JA, McKee M. Identification of an extensive system of nitric oxide-producing cells in the ciliary muscle and outflow pathway of the human eye. Invest Ophthalmol Vis Sci. 1995;36(9):17651773.

53. Swaminathan SS, Oh D-J, Kang MH, Rhee DJ. Aqueous outflow: segmental and distal flow. J Cataract Refract Surg. 2014;40(8):1263-1272.

54. Weinreb RN, Ong T, Scassellati Sforzolini B, et al. A randomised, controlled comparison of latanoprostene bunod and latanoprost $0.005 \%$ in the treatment of ocular hypertension and open angle glaucoma: the VOYAGER study. Br J Ophthalmol. 2015;99(6):738-745.

55. Kaufman PL. Latanoprostene bunod ophthalmic solution $0.024 \%$ for IOP lowering in glaucoma and ocular hypertension. Expert Opin Pharmacother. 2017;18(4):433-444.

56. Doganay S, Evereklioglu C, Turkoz Y, Er H. Decreased nitric oxide production in primary open-angle glaucoma. Eur J Ophthalmol. 2008;12(1):44-48.

57. Kang JH, Wiggs JL, Rosner BA, et al. Endothelial nitric oxide synthase gene variants and primary openangle glaucoma: interactions with sex and postmenopausal hormone use. Invest Ophthalmol Vis Sci. 2010;51(2):971-979.

58. Galassi F, Renieri G, Sodi A, Ucci F, Vannozzi L, Masini E. Nitric oxide proxies and ocular perfusion pressure in primary open angle glaucoma. Br J Ophthalmol. 2004;88(6):757-760. 


\section{Figures}

Figure 1: Effect of NO on facility in anterior segment cultures with intact TM

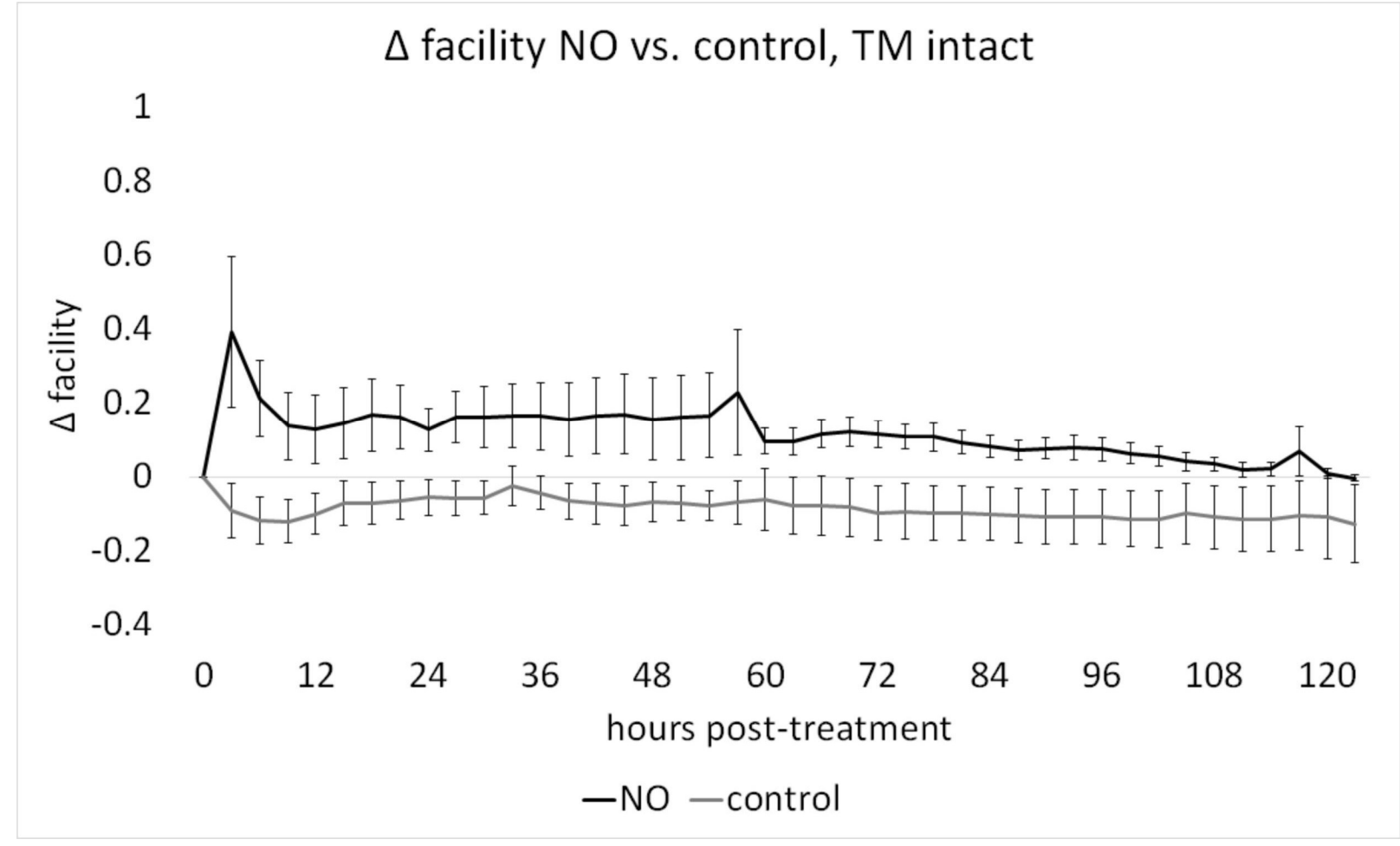

Figure 1. Effect of NO on facility in anterior segment cultures with intact TM. Outflow facility in NO remained above that of $C$ throughout the experiment. Change in facility for $C(-0.087 \pm 0.010 \mu \mathrm{L} / \mathrm{min} \cdot \mathrm{mmHg})$ was compared to NO $(0.113 \pm 0.011 \mu \mathrm{L} / \mathrm{min} \cdot \mathrm{mmHg})$ with a mixed-effects model and showed an NOmediated facility increase of $0.189 \pm 0.081 \mu \mathrm{L} / \mathrm{min} \cdot \mathrm{mmHg}(p=0.034, \mathrm{n}: \mathrm{NO}=8, \mathrm{C}=8)$. 
Figure 2: Effect of NO on facility in anterior segment cultures after TM ablation.

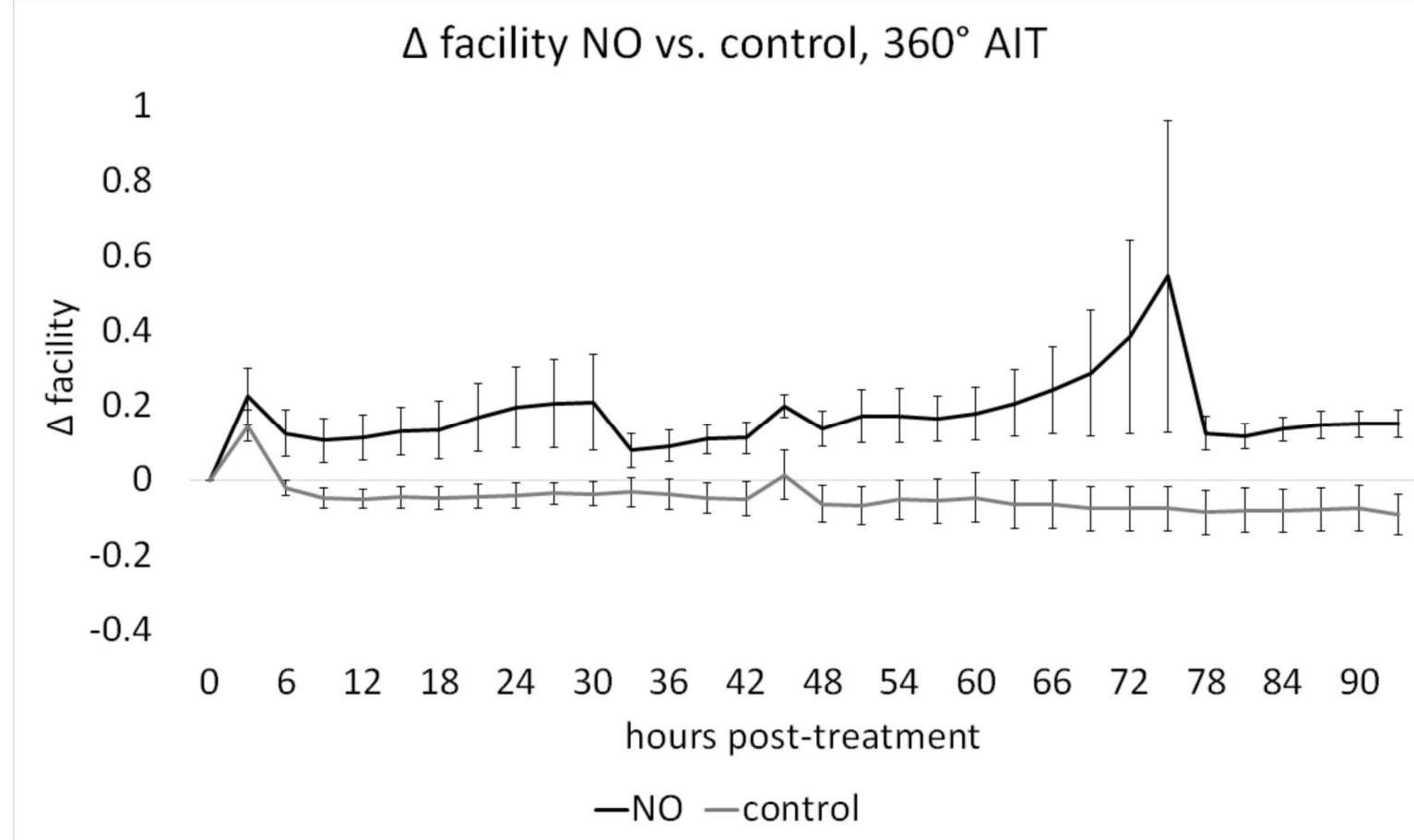

Figure 2. Effect of NO on facility in anterior segment cultures after TM ablation. Outflow facility in AIT-NO remained above that of AIT-C throughout the experiment. Post-treatment change in facility for AIT-NO $(0.178 \pm 0.021 \mu \mathrm{L} / \mathrm{min} \cdot \mathrm{mmHg})$ was compared to AIT-C $(-0.054 \pm 0.009 \mu \mathrm{L} / \mathrm{min} \cdot \mathrm{mmHg})$ with a mixed effects model and indicated an NO-mediated facility increase of $0.190 \pm 0.074 \mu \mathrm{L} / \mathrm{min} \cdot \mathrm{mmHg}(\mathrm{p}=0.023, \mathrm{n}$ : AlT-NO=7, AIT-C=8). 


\section{Figure 3: Rendering and alignment of adjacent outflow tract vessel volumes in 3D}

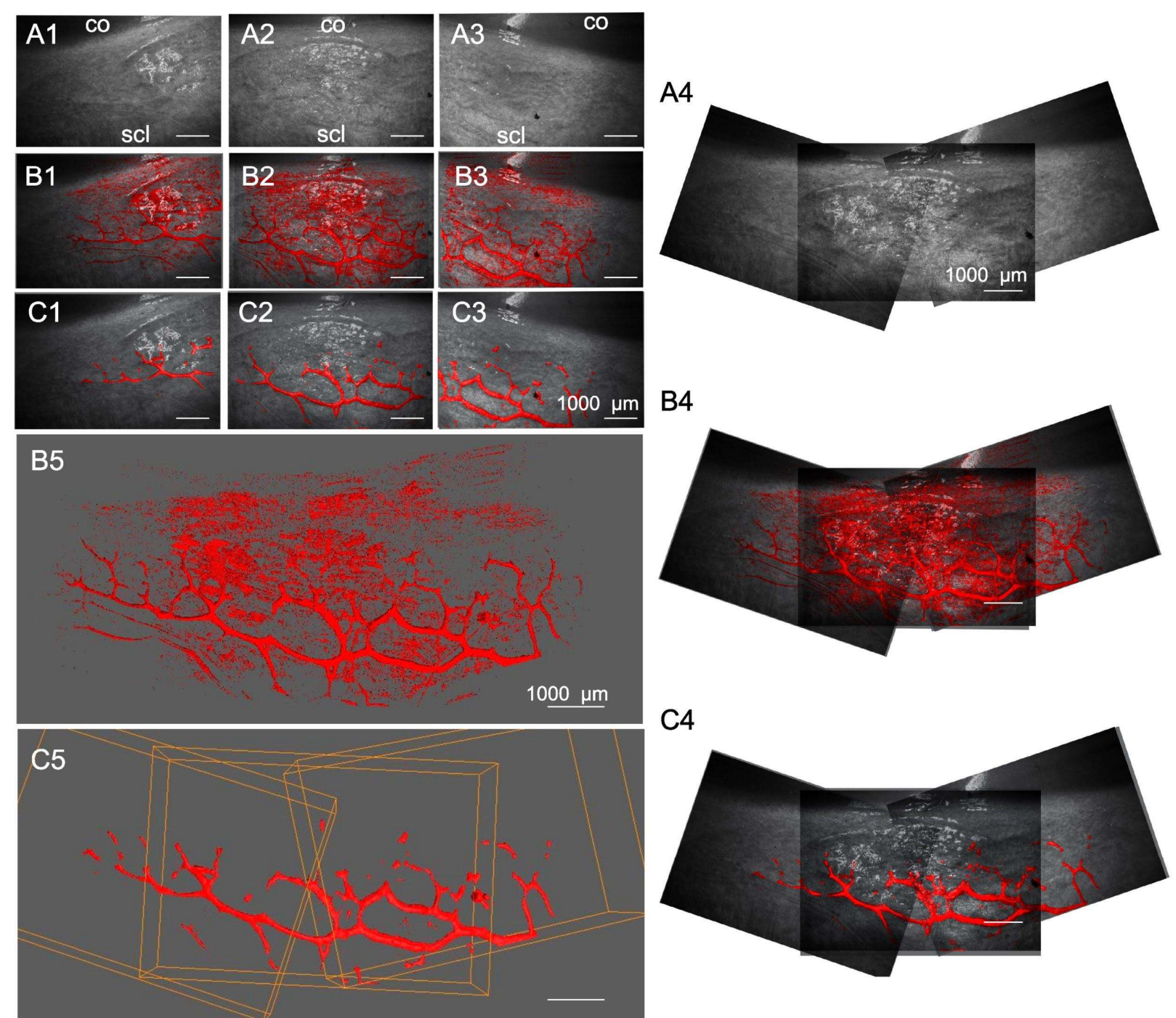

Figure 3: Rendering and alignment of adjacent outflow tract vessel volumes in 3D. Volume intensity projections (VIPS) (A1-3) of adjacent OCT scans with minimally processed (B1-3) and cleaned signal voids (C1-3) in overlay. A4-C4) 2D alignment of images in A-C, 1-3 fails to account for the curvature of the ocular surface, resulting in alignment artifacts. B5) surfaces in in B1-3, and C5) in C1-3 are aligned in 3D-space to neighboring scans without artifacts. Bounding box (orange) coordinates in $\mathrm{C} 5$ were conferred to respective scans in B5 to align them. co= cornea, scl= sclera. 


\section{Figure 4: Quantification of cross-sectional area pre-and post-treatment}

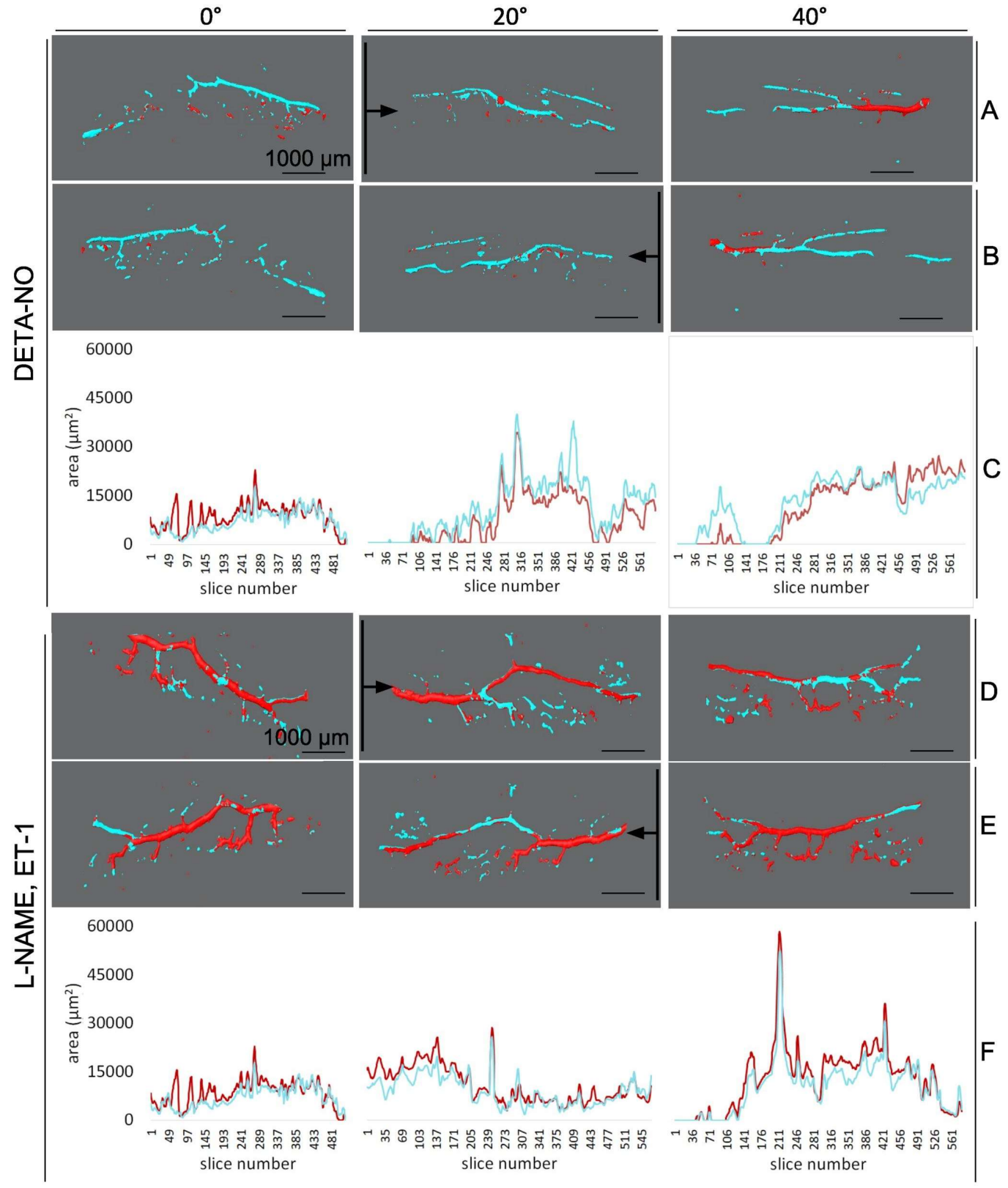

Figure 4: Quantification and of cross-sectional area pre- and post-treatment. Aligned and merged surface reconstructions taken at 0,20 , and $40^{\circ}$ with pre-treatment pseudocolored in red and post-treatment in cyan as viewed anteriorly $(A, D)$ and posteriorly $(B, E)$. Eyes treated with L-NAME + ET-1 showed a greater coverage with red throughout, indicating larger pre-treatment than post-treatment intraluminal space (constriction), and DETA-NO treated vessels show greater coverage with cyan, indicating a larger posttreatment than pre-treatment intraluminal space (dilation). Cross-sectional area (CSA) of virtual sections, made along the plane indicated by the black lines and in the direction of the black arrows, was calculated in silico. CSA is plotted throughout the length each sample (C, F). 
Figure 5: cross-sectional area of outflow tract vessels pre-and post-treatment

\section{CSA pre and post-treatment}

\section{0}

10000

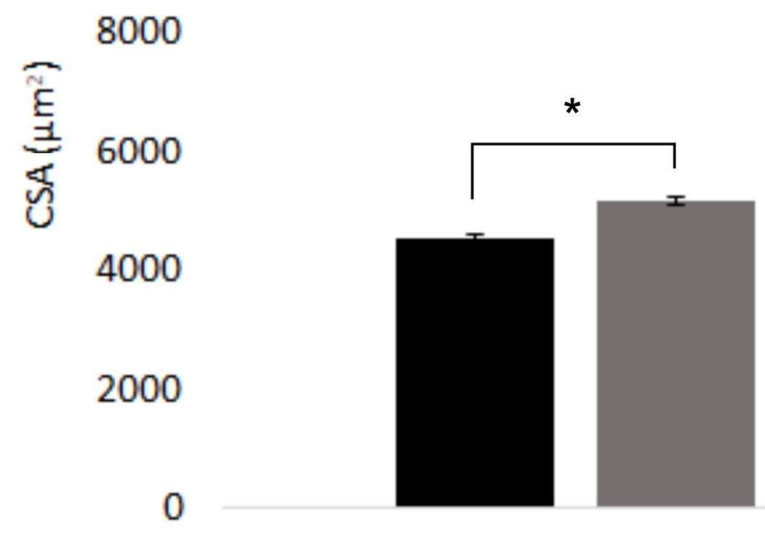

NO

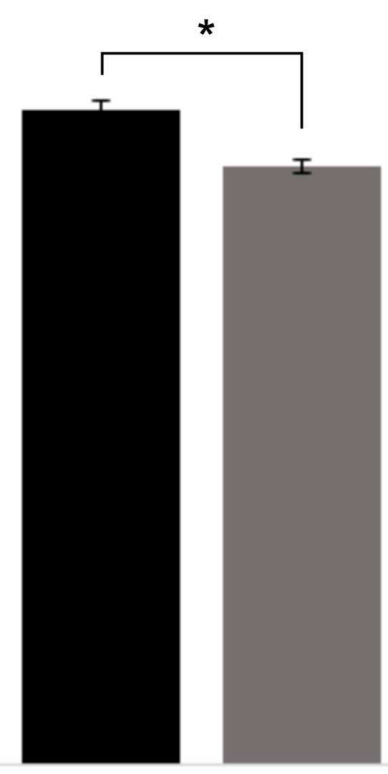

L-NAME ET-1

treatment

a pre-treatment $\quad$ post-treatment

$\mathrm{NO} \mathrm{N}=3$

L-NAME N=3

\begin{tabular}{l|c|c} 
& NO & L-NAME+ET-1 \\
\hline pre-treatment $\left(\mu \mathrm{m}^{2}\right)$ & $4540.1 \pm 59.3$ & $11002.2 \pm 132.1$ \\
\hline post-treatment $\left(\mu \mathrm{m}^{2}\right)$ & $5170.1 \pm 63.9$ & $10059 \pm 128.5$ \\
\hline $\begin{array}{l}\text { CSA as percentage of } \\
\text { baseline }\end{array}$ & $113.9 \%$ & $91.4 \%$ \\
\hline
\end{tabular}

Figure 5. Cross-sectional area of outflow tract vessels pre-and post-treatment. $\mathrm{N}=3$ eyes per treatment group. 13,146 cross-sections were analyzed in NO and 10,580 in L-NAME + ET-1. ${ }^{*} p \leq 0.001$, values reported as mean \pm standard error. 


\section{Figure 6: NO-induced focal dilation of outflow tract vessels.}

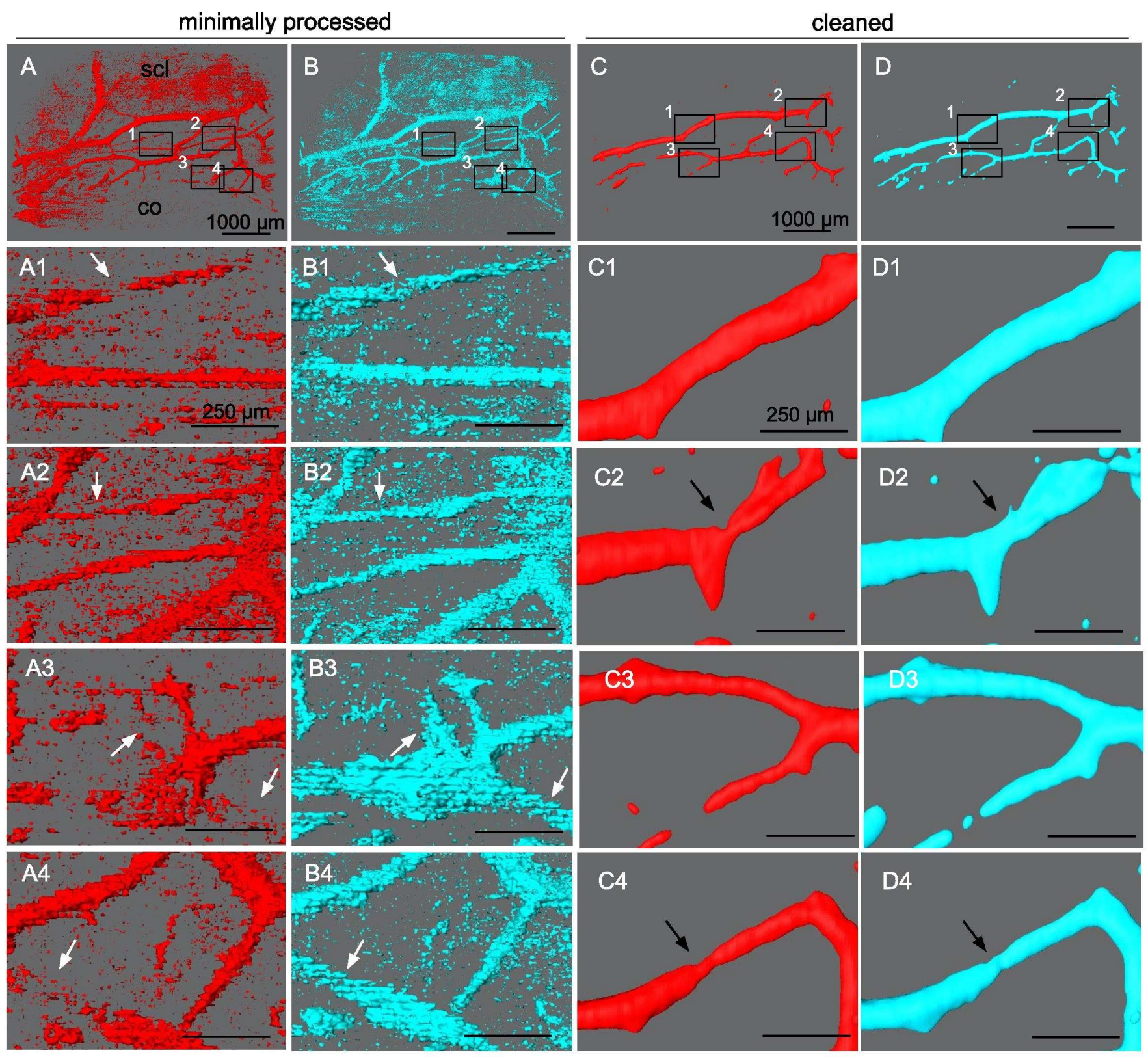

Figure 6: NO-induced focal dilation of outflow tract vessels. Outflow tract vessels pre- ( $A$ and $C$, red) and post-treatment (B and D, cyan) with DETA-NO vasodilator. Insets 1-4 in panels A-D show the location of magnified panels A1-D4. Dilation of outflow tract vessels can be seen in minimally processed reconstructions of SD-OCT signal voids (A4-B4). Outflow tract vessels can be seen to open at previously closed locations (white arrows), dilate at focal sites of high response (black arrows), and throughout their lengths. co=cornea, scl=sclera. 


\section{Figure 7: L-NAME + ET-1-induced focal constriction of outflow tract vessels}

minimally processed
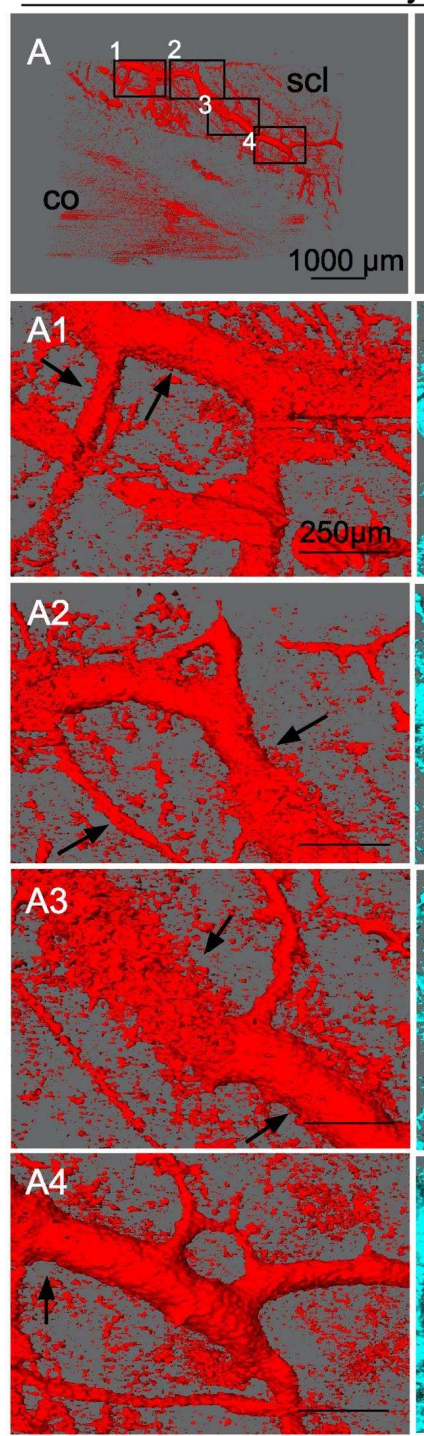
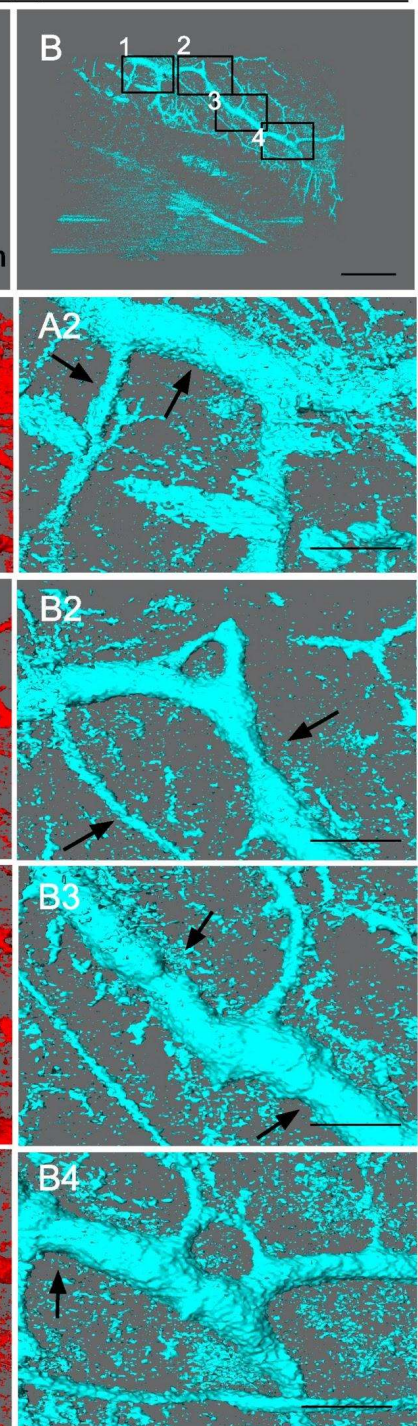

cleaned
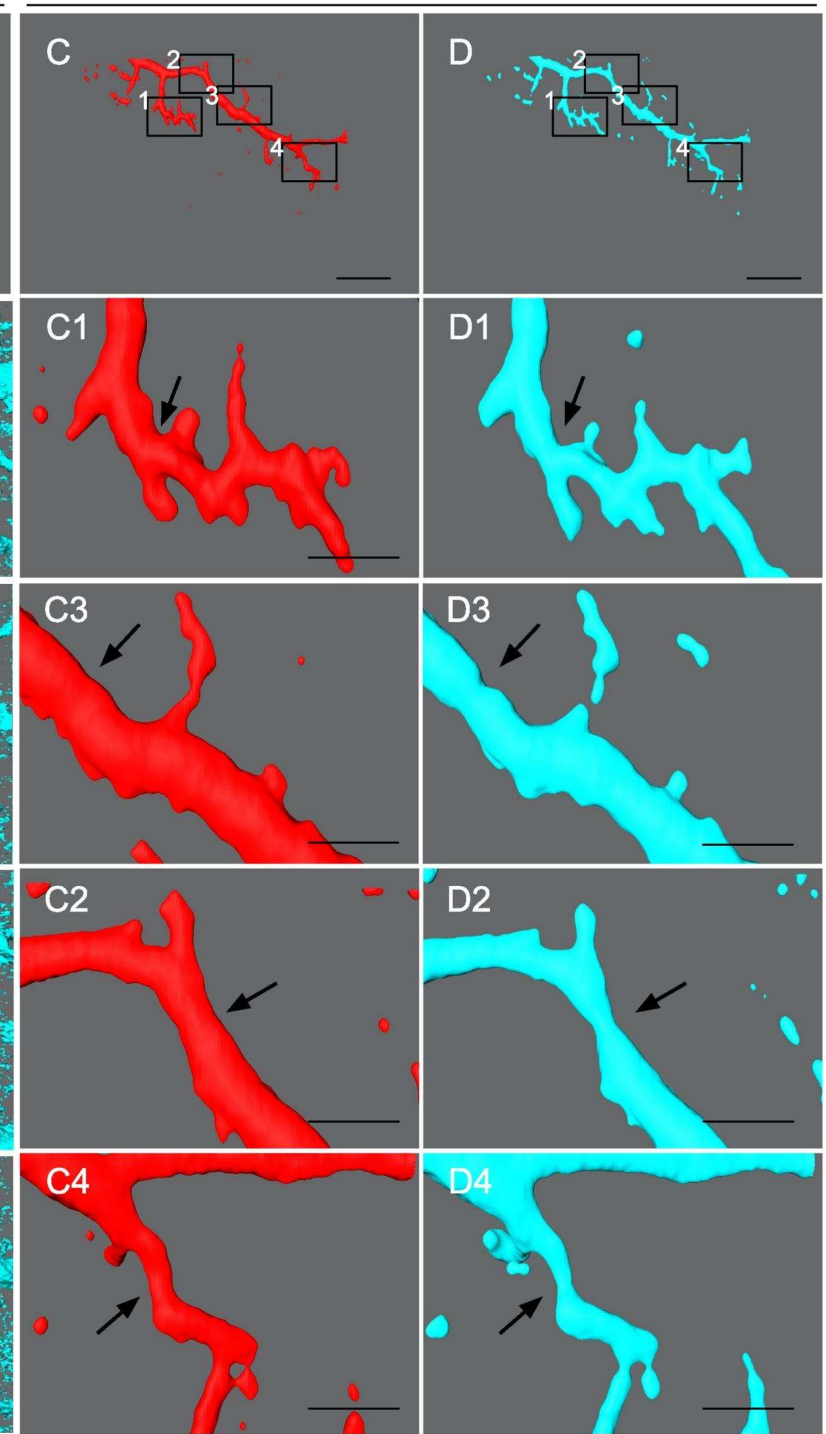

Figure 7: L-NAME + ET-1-induced focal constriction of outflow tract vessels. Outflow tract vessels pre- (red) and post-treatment (cyan) with L-NAME and ET-1 vasoconstrictors. Constriction of outflow tract vessels can be seen in projections of minimally processed scans (A-B4) as well in as cleaned scans used for quantitative slice-by-slice comparison (C-D4). Insets 1-4 in panels A-D indicate the location of the magnified panels A1D4. Outflow tract vessels can be seen to constrict or collapse at focal sites of high response (black arrows), and constrict throughout their lengths. $\mathrm{co}=\mathrm{cornea}$, scl=sclera 


\section{Supplementary Material}

\section{Supplementary Figure 1: SD-OCT imaging setup}
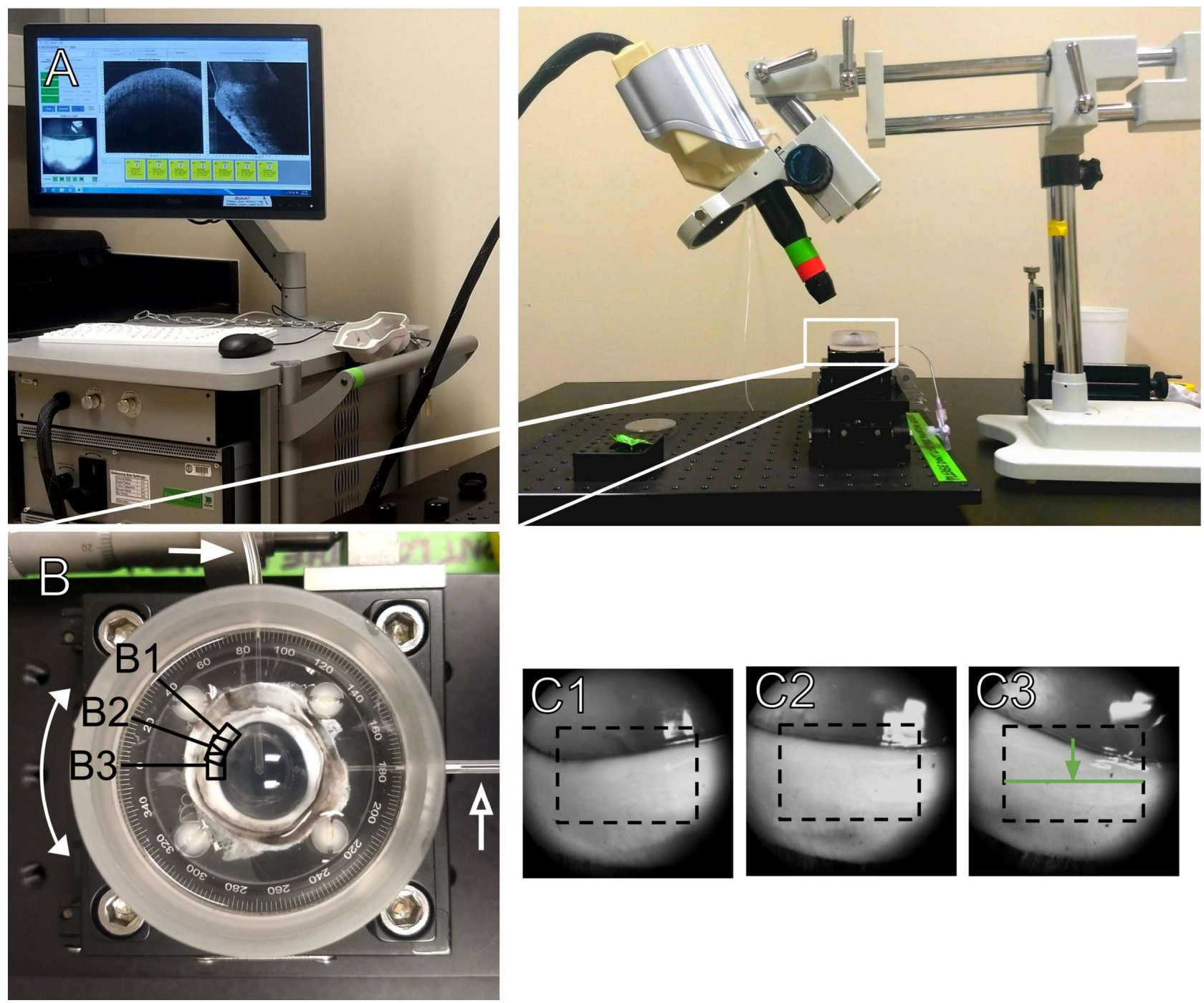

Supplementary Figure 1: SD-OCT imaging setup. A) Anterior segments were affixed to a rotatable stage (white box) under the scanning arm of an R2210 Envisu SD-OCT. Before image capture, limbal tissue was visualized in Bioptigen InvivoVue software through a $10 \mathrm{~mm}$ telecentric lens. B) Anterior segment in its perfusion dish, the infusion line (straight, closed arrow), the outlet (open arrow), direction of dish rotation (curved arrow) and the location of scans along the limbus (black boxes B1-B3). C1-C3) Representative sample areas corresponding with locations B1-B3, each $20^{\circ}$ apart. The plane and direction of B-scan capture is indicated in green. Scans were acquired over a sample area $6.0 \mathrm{~mm}$ long, $4.0 \mathrm{~mm}$ wide, and $1.6 \mathrm{~mm}$ deep with $1000 \times 600 \times 1024$ pixels. 
Supplementary Table 1: CSA pre-and post-treatment

\begin{tabular}{|l|c|c|c|}
\hline sample ID & $\begin{array}{c}\text { pre-treatment CSA } \\
\left(\boldsymbol{\mu \mathbf { m } ^ { 2 } )} \pm \mathbf{S E M}\right.\end{array}$ & $\begin{array}{c}\text { post-treatment CSA } \\
\left(\boldsymbol{\mu \mathbf { m } ^ { 2 } )} \pm \mathbf{S E M}\right.\end{array}$ & $\boldsymbol{\Delta}$ CSA (\% change) \\
\hline eye 1, DETA-NO & $2959.2 \pm 89.6$ & $3346.0 \pm 95.8$ & $386.7 \pm 44.9(13.1)$ \\
\hline eye 2, DETA-NO & $3406.4 \pm 117.1$ & $3455.4 \pm 124.5$ & $49.0 \pm 44.6(1.4)$ \\
\hline eye 3, DETA-NO & $6157.6 \pm 86.8$ & $7277.6 \pm 90.2$ & $1120.0 \pm 39.9(18.2)$ \\
\hline eye 4, L-NAME + ET-1 & $14818.2 \pm 286.5$ & $14399.6 \pm 287.0$ & $-418.6 \pm 84.0(-2.8)$ \\
\hline eye 5, L-NAME + ET-1 & $10103.0 \pm 173.4$ & $8329.8 \pm 142.5$ & $-1773.2 \pm 61.7(-17.6)$ \\
\hline eye 6, L-NAME + ET-1 & $8121.5 \pm 179.2$ & $7475.9 \pm 174.4$ & $-645.6 \pm 54.2(-7.9)$ \\
\hline
\end{tabular}

values reported as mean \pm standard error 